\title{
Hypermucoviscosity in Clinical Isolates of Klebsiella pneumoniae Correlates with High Multiple Antibiotic Resistance (MAR) Index
}

\author{
Uzal Umar1*, Sandra Anagor'1, Abdullahi Aliyu1, Abdulmumini Ibrahim Suleiman² \\ ${ }^{1}$ Department of Biological Sciences, Abubakar Tafawa Balewa University, Bauchi, Nigeria \\ ${ }^{2}$ Department of Clinical Microbiology, Abubakar Tafawa Balewa University Teaching Hospital, Bauchi, Nigeria \\ Email: "uumar2@atbu.edu.ng
}

Received 31 March 2016; accepted 18 July 2016; published 21 July 2016

Copyright (C) 2016 by authors and Scientific Research Publishing Inc.

This work is licensed under the Creative Commons Attribution International License (CC BY).

http://creativecommons.org/licenses/by/4.0/

(c) (i) Open Access

\section{Abstract}

Klebsiella pneumoniae is an opportunistic pathogen of medical importance and the capsule and mucoid phenotype in this organism are considered as requisite virulence determinants. A total of 62 clinical samples from ATBUTH were collected and screened for $K$. pneumoniae. The isolates were identified using standard tests for this organism. The string test was used to detect the mucoid (hypermucoviscous) phenotype and the antimicrobial susceptibility test to 10 antibiotics was carried out with the disk diffusion technique after standardizing inoculum. A $K$. pneumoniae prevalence of $24 \%(15 / 62)$ was obtained of which $47 \%(7 / 15)$ were mucoid (hypermucoviscous) and $53 \%(8 / 15)$ were non-mucoid. Colonial sizes of the two strains do not reveal any significant differences in growth fitness of the strains. On blood agar, the mucoid and non-mucoid strains had a mean colonial size \pm standard deviations of $4.41 \pm 0.58 \mathrm{~mm}$ and $4.27 \pm 0.42 \mathrm{~mm}$ respectively. The antibiotic susceptibility rate showed that the mucoid strains compared to the non-mucoid were more resistant to nine out of $\mathbf{1 0}$ antibiotics. The mucoid strains were outrightly resistant to augmentin, amoxicillin, septrin, sparfloxacin and perfloxacin. The non-mucoid strains showed no complete resistant to any antibiotic tested but had a higher resistant rate to chloramphenicol only. The Multiple Antibiotic Resistance (MAR) index shows the themucoid strains with a high MAR index range of 0.7 - 1.0 with a median MAR index of 0.8 , while the non-mucoid strains had a MAR index of $0.2-0.8$ with a median MAR index of 0.35 . The data suggest that the mucoid phenotype could be associated with extrachromsomal element(s) carrying resistance genes to antibiotics and that these extrachromosomal elements may not harbour resistance determinants to chloramphenicol. Furthermore, the extrachromosomal elements bearing the mucoid phenotype and the resistance elements in the mucoid strains do not significantly impact on the fitness of the cognate strain. Whether these phenotype and resistances that had no fitness cost to the bacterium could significantly affect the virulence of the bacteria in vivo remains to be investigated.

${ }^{*}$ Corresponding author.

How to cite this paper: Umar, U., Anagor, S., Aliyu, A. and Suleiman, A.I. (2016) Hypermucoviscosity in Clinical Isolates of Klebsiella pneumoniae Correlates with High Multiple Antibiotic Resistance (MAR) Index. Open Journal of Medical Microbiology, 6, 97-103. http://dx.doi.org/10.4236/ojmm.2016.63013 


\section{Keywords}

\section{Hypermucoviscousity, PLA, Phagocytosis, Siderophores, Virulence}

\section{Introduction}

Klebsiella pneumoniae is a gram negative bacteria and an opportunistic pathogen. In humans K. pneumoniae is an important species of medical importance of the genus Klebsiella and is found as saprophyte in the nasopharynx and in the gastrointestinal tract. K. pneumoniae causes suppurative infections, bacteremia and septicaemia. The bacteria accounts for a great majority of hospital associated infections in neonates, immunocompromised patients, patients undergoing respiratory therapies and patients hospitalized in urology and burn wards. Example of serious community associated infections due to K. pneumoniae is Friedlander's pneumonia, a severe form of lobar pneumonia with high mortality, and in some countries the organism is the leading cause of pneumonia. Recently K. pneumoniae invasive community acquired pyogenic liver abscess (PLA) sometimes complicated by endophthalmitis or meningitis emerged in Taiwan and other Asian countries as well as other continents [1].

$K$. pneumoniae elaborates a number of virulence factors that contribute to pathogenesis; these virulence factors are broadly grouped as fimbrial and non-fimbrial adhesins, siderophores, somatic ("O") antigens and capsu$\operatorname{lar}($ " $\mathrm{K}$ ") antigens. The capsule is regarded as the dominant virulence factor and consists of an elaborate layer of surface-associated polysaccharides the composition of which is strain dependent [2]. Some encapsulated Klebsiella species form glistening mucoid colonies of viscid consistency. The contribution of the mucoid phenotype to pathogenesis has been demonstrated in several models: in general, the mucoid isolates are more resistant to phagocytosis, less sensitive to serum killing and more virulent in animal studies. Also highlighted is the fact that phenotypic switching between mucoid and non-mucoid morphology under different in vivo conditions can also influence the host immune response [3].

The gene $r m p$ A (regulator of the mucoid phenotype) encoding the hypermucoviscous (HV) phenotype was described on a $180-\mathrm{kb}$ plasmid. Two phenotypes that enhance the virulence of the organism are associated with the presence of the $180-\mathrm{kb}$ plasmid: namely production of siderophores and the mucoid phenotype. To confirm the presence of the gene encoding the mucoid phenotype on a plasmid, the phenotype could be lost by curing of the mucoidstrain [4]. Similarly, the HV phenotype in K. pneumoniae is said to be associated to novel virulence genes such as magA [4], cps [5] wbs cluster [4]. Because so many genetic loci are associated with the hypermucoviscous phenotype, the critical role of the phenotype in K. pneumoniae pathogenesis remained unresolved [5].

$K$. pneumoniae isolates like other enterobacteriaceae are increasingly resistant to multiple antimicrobial agents including aminoglycosides and quinolones and the 3rd generation cephalospsorins such as ceftazidime [6]. Resistance to the third generation cephalosporins is mediated by the production of Extended Spectrum Beta Lactamases (ESBLs). Though ESBLs phenotype is widespread in gram negative bacteria, $K$. pneumoniae is the most common species producing ESBLs [7]. ESBLs were first reported in Germany in K. pneumoniae in 1983 but report of its prevalence is increasing worldwide sometimes within hospitals and between countries [8]. Genes encoding ESBLs are usually located on transferable plasmids that may also carry other resistance determinants such as those for resistance to aminoglycosides, tetracycline, chloramphenicol, trimethoprim and sulfonamides [9]. Most outbreaks of epidemic strains the major means of spread of ESBLs are limited to areas where high risk patients are cared for such as neonates, burns patient etc. The mechanism of ESBL resistance has been implicated in the virulence of the organism by decreasing/increasing resistance to phagocytosis and imposing a metabolic fitness cost [10]. We sought to investigate if strains with the potential for virulence could be disseminated within the community and whether there was a correlation with virulent strains and antibiotic resistance determinants in the community.

\section{Materials and Methods}

\subsection{Isolation and Identification of Klebsiella pneumonia (Clinical Samples Processing)}

A total number of sixty-two (62) non-duplicate urine and wound swabs clinical specimens submitted to the microbiology laboratory Abubakar Tafawa Balewa University Bauchi Nigeria were obtained and cultured on 
MacConkey agar and incubated aerobically for 24 hours at $37^{\circ} \mathrm{C}$. Bacteria were identified as K. pneumoniae on the basis of standard clinical microbiological methods such as colonial characteristics on MacConkey agar after incubation aerobically for 24 hours at $37^{\circ} \mathrm{C}$, lactose fermentation on MacConkey agar and Gram stain reaction. Suspected K. pneumoniae isolated were further subjected to biochemical test such as urease production, fermentation of sugars in triple sugar Iron without $\mathrm{H}_{2} \mathrm{~S}$ production and catalase test. K. pneumoniae isolates are Gram negative, lactose fermenting, catalase positive and ferment glucose, lactose and sucrose. Discrete colonies were obtained, labeled and streaked on a nutrient agar slant for preservation. Nutrient agar Slants were maintained at $4^{\circ} \mathrm{C}$ until further used.

\subsection{Determination of Growth Rate of Strains}

The growth rate of the strains were determined by streaking each strain to purity on MacConkey agar plates and incubated aerobically at $37^{\circ} \mathrm{C}$ for $16 \mathrm{~h}$. The colonial diameters for ten (10) randomly selected colonies were measured in millimeters and results recorded. Two independent experiments were carried out for each strain and results averaged. The mean and standard deviations of strains with the mucoid (hypermucoviscous) and the non-mucoid phenotype was plotted.

\subsection{Determination of Mucoid Phenotype (Hypermucoviscousity)}

The string test where a standard bacteriological loop is used to stretch a mucoviscous string from the bacterial colony was utilized to determine the mucoid phenotype. Stock strains from preserved slants were streaked to purity on $5 \%$ sheep blood agar plates and incubated at $37^{\circ} \mathrm{C}$ overnight $(16 \mathrm{~h})$. A standard bacteriological loop was used to touch a colony and stretched to generate a mucoviscous string. The formation of a viscous string > $10 \mathrm{~mm}$ depicts a hypermucoviscous phenotype was regarded as a positive confirmation of the hypermucoviscousity (HV) phenotype of the strain [3]. The experiment was carried out twice and results averaged with standard deviations.

\subsection{Determination of Antibiotic Susceptibility}

Stock strains were obtained and streaked to purity on nutrient agar plates and incubated aerobically at $37^{\circ} \mathrm{C}$. Discrete colonies were picked and emulsified in $0.45 \%(\mathrm{w} / \mathrm{v})$ sterile aqueous normal saline. The suspension optical density is standardized to a McFarland density of 0.5 (equivalent to CFU $/ \mathrm{ml}$ ) with the aid of a Densi$\mathrm{Chek}^{\mathrm{TM}}$ densitometer (bioMerieux, USA) apparatus. The suspensions were used within 15 minutes of standardization. Dry, sterile, absorbent cotton wool was dipped into the standardized suspension and excess moisture drained by pressing the wet cotton wool against the walls of the test tube. The cotton wool was then used to streak the surface of a Mueller-Hinton agarplate, which was earlier poured to a uniform depth of $5 \mathrm{~mm}$ and dried in the incubator for 15 minutes to reduce excess moisture. The inoculated plates were allowed to stand for 5 minutes and the antibiotic susceptibility disc is placed on the inoculated Mueller-Hinton agar plate. The plates were then incubated aerobically at $37^{\circ} \mathrm{C}$ for $16 \mathrm{~h}$. After overnight incubation the zone of inhibitions were measured with the aid of a meter rule in two directions across each inhibition zone and the results averaged and recorded. The experiment was carried out twice for each strain and the results averaged and recorded as the zone of inhibition of the antibiotic for each strain. Modified EUCAST 2007 guidelines for interpretative criteria for susceptibility to antibiotics were adopted; the isolates were thus designated susceptible or resistant [11].

\section{Results}

Prevalence of K. pneumoniae in clinical specimens; prevalence of mucoid (Hypermucpviscous) Strains of $K$. pneumoniae; Growth Rate (Fitness) of Mucoid (Hypermucoviscous) and Non-Mucoidal strains: A K. pneumoniae prevalence of $24 \%(15 / 62)$ was obtained from clinical samples processed (data not shown), of which about $47 \%(7 / 15)$ and 53\% (8/15) were mucoidal (HV) and non-mucoidal strains respectively (Figure 1). The biological fitness of the two strains were compared using mean \pm standard deviations of their colonial size on blood agar, the mucoidal strains had the average size and deviations of $4.21 \mathrm{~mm} \pm 0.58 \mathrm{~mm}$ and the non-mucoidal strains $4.27 \mathrm{~mm} \pm 0.42 \mathrm{~mm}$ (Figure 2). The means were compared using student t-test and no significant difference was observed $(\mathrm{p}<0.05)$ between the colonial sizes of mucoid and non-mucoid strains.

Antibiotic susceptibilities of mucoid and non-mucoid strains; the antibiotic susceptibilities of all the strains 


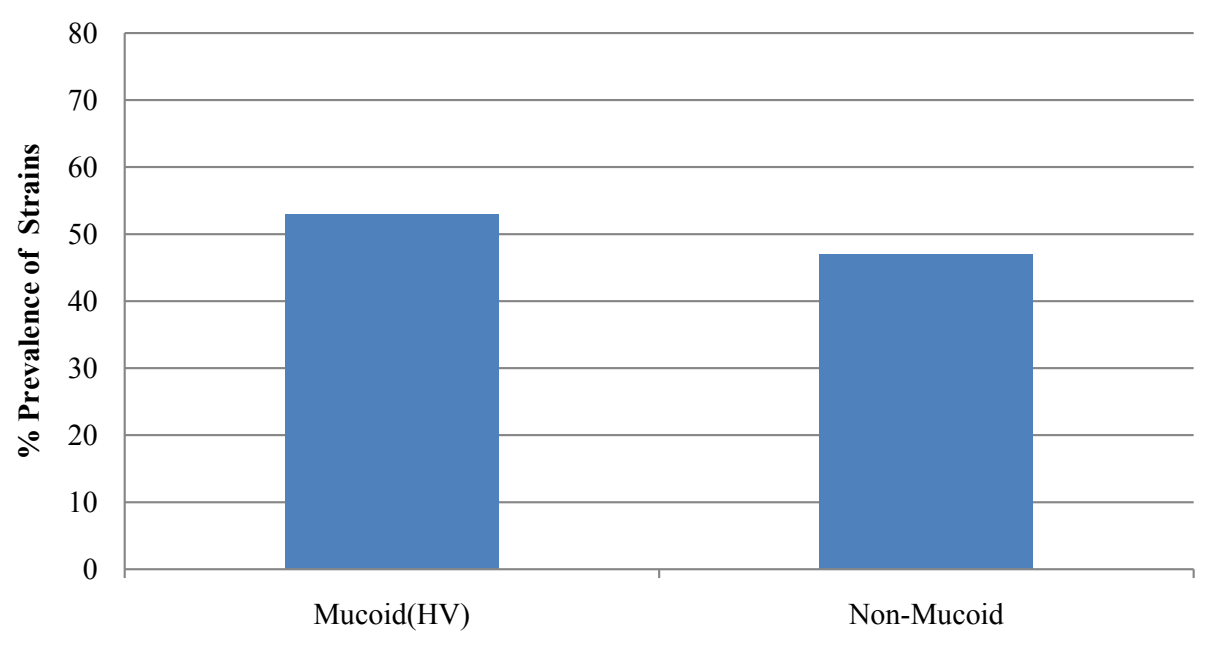

Strains Phenotype

Figure 1. Prevalence of Mucoid (hypermucovscous) and non-Mucoid strains of K. pneumoniae from clinical specimens. Isolated $K$. pneumoniae were streaked on $5 \%$ sheep blood agar, incubated aerobically at $37^{\circ} \mathrm{C}$ for $16 \mathrm{~h}$. After overnight incubation the colony was touched with the aid of plastic wireloop raised to form a string and thus identified as mucoid (hv) or non-mucoid.

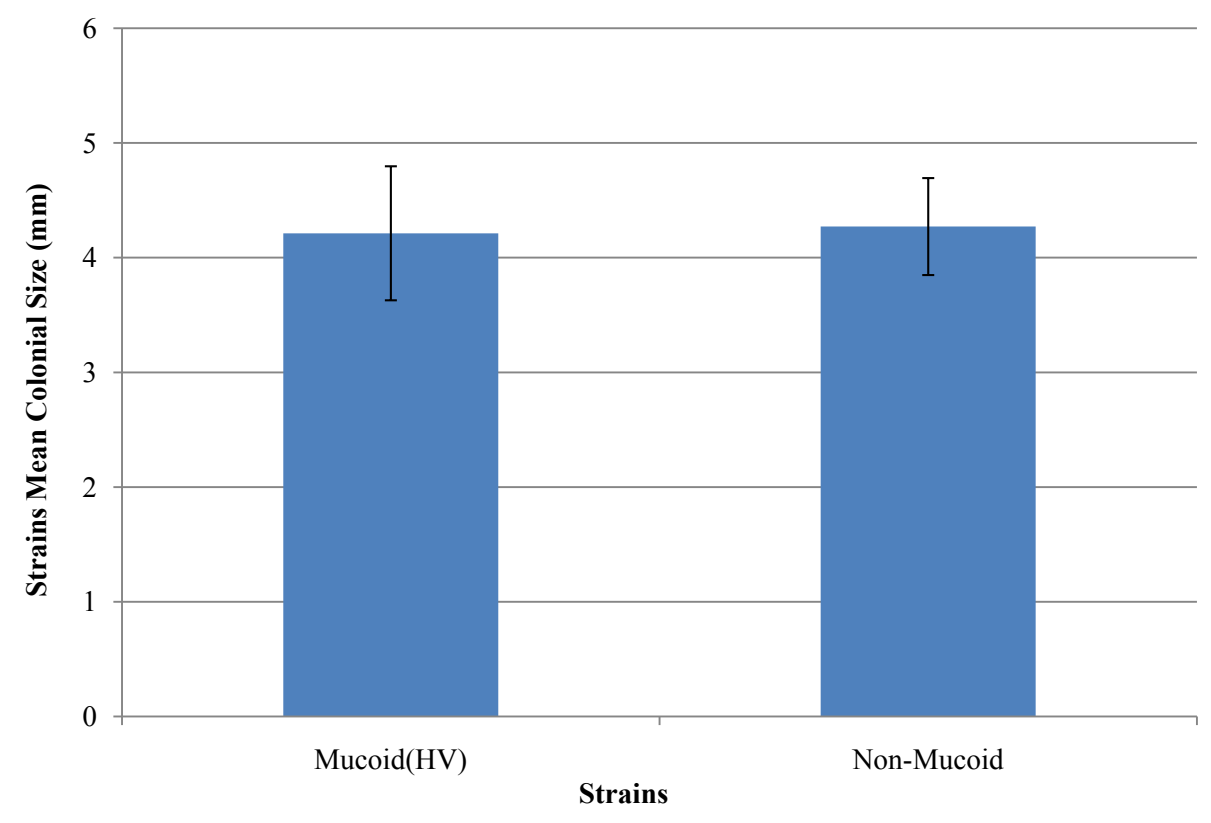

Figure 2. Growth rate of mucoid and non-mucoid strains assessed from colonial sizes. The strain is inoculated unto McConkey agar, incubated aerobically for $16 \mathrm{~h}$ at $37^{\circ} \mathrm{C}$. The diameters of 10 randomly selected colonies for each strain was measured and averaged. The means of the colonies for each strain was further averaged and standard deviation was obtained and plotted. The experiment was carried out twice.

tested against 10 antibiotics showed high resistance rate to sparfloxacin (93\%), perfloxacin (93\%), augmentin $(80 \%)$, trimethoprim + sulfamethaxazole (73\%) amoxicillin $(60 \%)$ ciprofloxacin $(60 \%)$ and streptomycin $(53 \%)$. The isolates were more sensitive to gentamicin $(60 \%)$ azithromycin (60\%), chloramphenicol (53\%) (Table 1). When the strains were separated into the mucoid and non-mucoid strains their antibiotic susceptibility pattern shows a clear distinction. The mucoid strains were completely resistant to five antimicrobials namely amoxicillin, augmentin ( $\beta$-Lactams), sparfloxacin, perfloxacin (quinolones) and septrin (a trimethoprim + sulphonamide), 
furthermore, they were resistant to azithromycin (86\%), streptomycin (86\%), ciprofloxacin (86\%) and gentami$\operatorname{cin}(71 \%)$. While the non-mucoid strains were resistant to perfloxacin $(87.5 \%)$, sparfloxacin $(87.5 \%)$, augmentin $(62.5 \%)$ and chloramphenicol (62.5\%). A high antibiotic resistance rate could be detected more often in mucoid (hv) strains than non-mucoid strains; augmentin $(100 \%$ vs. $25 \%$; p < 0.07973$)$ amoxicillin $(100 \%$ vs. $62.5 \%$; $p$ $<0.22859)$, trimethoprim + sulfamethaxazole $(100 \%$ vs. $50 \%$; p < 0.19982$)$, azithromycin $(86 \%$ vs. $12.5 \%$; $<<$ 0.05281 ), chloramphenicol ( $29 \%$ vs. $62.5 \%$; p < 0.39765), streptomycin ( $86 \%$ vs. $25 \%$; p < 0.27932$)$, perfloxacin $(100 \%$ vs. $87.5 \%$; p < 0.69967$)$, sparfloxacin $(100 \%$ vs. $87.5 \%$; $<0.69967)$, gentamycin $(71 \%$ vs. $25 \%$; $<0.53283$ ) ciprofloxacin ( $86 \%$ vs. $37.5 \% ; \mathrm{p}<0.14655)$ (Table 2); Multiple Antibiotic Resistance (MAR) index of mucoid (hv) and non-mucoidal Strains (Table 3). Resistance to single antibiotic was not found among the strains, though 10 antibiotics were tested, the antibiotics belong to six classes of antimicrobials; $\beta$-Lactams (2), sulphonamide (1), aminoglycosides (3), chloramphenicol (1) and quinolones (3). The mucoid (hv) strains had a high MAR index with a range of $0.7-1.0$ with a median MAR index of 0.8 . In contrast the non-mucoid strains showed a broad MAR index of $0.2-0.8$ with a median MAR index of 0.35 .

Table 1. Antibiotic susceptibilities of clinical isolates of K. pneumonia.

\begin{tabular}{cccccc}
\hline Antibiotic & Modified EUCAST & \multicolumn{3}{c}{ Antibiotic Susceptibility of Isolates } \\
\cline { 3 - 6 }$($ Disc Potency) & Breakpoints (mm) S/R & Total No. of Isolates & No. Sensitive (\%) & No. Resistant (\%) & p-value \\
\hline Azithromycin $(10 \mu \mathrm{g})$ & $>14 /<14$ & 15 & $9(60)$ & $6(40)$ & 0.35363 \\
Amoxicillin $(30 \mu \mathrm{g})$ & $>14 /<14$ & 15 & $6(40)$ & $9(60)$ & 0.65114 \\
Trimethoprim + & $>15 /<15$ & 15 & $4(27)$ & $11(73)$ & 0.13234 \\
Sulfamethaxazole $(30 \mu \mathrm{g})$ & $>15 /<15$ & 15 & $7(47)$ & $8(53)$ & 0.96009 \\
Streptomycin $(10 \mu \mathrm{g})$ & $>16 /<16$ & 15 & $9(60)$ & $6(40)$ & 0.35363 \\
Gentamicin $(10 \mu \mathrm{g})$ & $>17 /<17$ & 15 & $8(53)$ & $7(47)$ & 0.40481 \\
Chloramphenicol $(30 \mu \mathrm{g})$ & $>19 /<19$ & 15 & $3(20)$ & $12(80)$ & 0.12434 \\
Augmentin $(30 \mu \mathrm{g})$ & $>21 /<21$ & 15 & $6(40)$ & $9(60)$ & 0.48004 \\
Ciprofloxacin $(10 \mu \mathrm{g})$ & $>21 /<21$ & 15 & $1(7)$ & $14(93)$ & 0.00682 \\
Sparfloxacin $(10 \mu \mathrm{g})$ & $>24 /<24$ & 15 & $1(7)$ & $14(93)$ & 0.00682 \\
Perfloxacin $(10 \mu \mathrm{g})$ & & & & \\
\hline
\end{tabular}

NB: The diameter of the antibiotic disc used was $8 \mathrm{~mm}$.

Table 2. Resistances of mucoid (hypermucoviscous) and non-mucoid K. pneumoniae clinical strains.

\begin{tabular}{cccc}
\hline Antibiotics & \multicolumn{2}{c}{ Strains } \\
(Disc Potency) & $\begin{array}{c}\text { Mucoid (\%) } \\
n=7\end{array}$ & $\begin{array}{c}\text { Non-Mucoid (\%) } \\
n=8\end{array}$ & p-value \\
\cline { 2 - 4 } Azithromycin $(10 \mu \mathrm{g})$ & 86 & 12.5 & 0.05281 \\
Amoxicillin $(30 \mu \mathrm{g})$ & 100 & 25 & 0.22859 \\
Trimethoprim + & 100 & 50 & 0.19982 \\
Sulfamethaxazole $(30 \mu \mathrm{g})$ & 86 & 25 & 0.27932 \\
Streptomycin $(10 \mu \mathrm{g})$ & 71 & 25 & 0.53283 \\
Gentamicin $(10 \mu \mathrm{g})$ & 29 & 62.5 & 0.39765 \\
Chloramphenicol $(30 \mu \mathrm{g})$ & 100 & 62.5 & 0.07873 \\
Augmentin $(30 \mu \mathrm{g})$ & 86 & 37.5 & 0.14655 \\
Ciprofloxacin $(10 \mu \mathrm{g})$ & 100 & 87.5 & 0.69967 \\
Sparfloxacin $(10 \mu \mathrm{g})$ & 100 & 87.5 & 0.69967 \\
Perfloxacin $(10 \mu \mathrm{g})$ & & & \\
\hline
\end{tabular}


Table 3. Multiple Antibiotic Resistance (MAR) index of mucoid (hypermucoviscous) and non-mucoid K. pneumoniae strains.

\begin{tabular}{cccl}
\hline MAR Index & $\begin{array}{c}\text { Mucoid } \\
\text { Strains }(\%)\end{array}$ & $\begin{array}{c}\text { Non-Mucoid } \\
\text { Strains (\%) }\end{array}$ & \multicolumn{1}{c}{ Antibiotic Resistance Pattern } \\
\hline 0.1 & $0(0)$ & $0(0)$ & Nil \\
0.2 & $0(0)$ & $1(12.5)$ & .AUG., CML. \\
0.3 & $0(0)$ & $2(25.0)$ & .AUG., PFC., SFC. \\
0.4 & $0(0)$ & $1(12.5)$ & .AUG., PFC., SFC., CFC. \\
0.5 & $0(0)$ & $1(12.5)$ & .AUG., PFC., SFC., CML., STX. \\
0.6 & $0(0)$ & $0(0)$ & Nil \\
0.7 & $1(14.3)$ & $1(12.5)$ & .AUG. AMX., PFC., SFC., STX., CFC., STM. \\
& & & $\begin{array}{l}\text {.AUG., AMX, PFC., SFC., STX., CFC., GEN., AZC. } \\
\text {.AUG., AMX., PFC., SFC., STX., CFC., CML., AZC. }\end{array}$ \\
0.8 & $3(42.9)$ & $2(25.0)$ & $\begin{array}{l}\text {.AUG. AMX., PFC., SFC., STX., GEN., AZC., CML. } \\
\text {.AUG. AMX., PFC., SFC., STX., STM., GEN., CML. }\end{array}$ \\
& & & .AUG. AMX, PFC., SFC., STX., CFC., STM., AZC. \\
1.0 & $2(28.5)$ & $0(0)$ & .AUG., AMX., PFC., SFC., STX., CFC., GEN., AZC., STM. \\
Total & $1(14.3)$ & $0(0)$ & .AUG., AMX., PFC., SFC., STX., CFC., GEN., AZC., STM, CML. \\
\hline
\end{tabular}

Key: AUG.-Augmentin, AMX.-Amoxicillin, CML.-Chloramphenicol, PFC.-Perfloxacin, SFC.-Sparfloxacin, CFC.-Ciprofloxacin, STX.Septrin, STM.-Streptomycin, GEN.-Gentamycin and AZC.-Azithromycin.

\section{Discussion}

The two samples (urine and wound swabs) screened are known to be a major source of $K$. pneumonia (Cosgrove, 2006). The mucoid (hypermucoviscous) phenotype is widespread among $K$. pneumoniae, more commonly associated with community acquired $K$. pneumonia bacteraemia (CA-KpB) (Lee et al., 2006) and a dominant feature of purulent infections [5].

Comparison of the means + standard deviations of the colonial sizes of the mucoid (hv) and the non-mucoid strains suggests there is no fitness cost in the phenotypic switching between the mucoid and non-mucoid phenotype. Even though antibiotic resistance determinant and the $r m p \mathrm{~A}$, regulators of the mucoid phenotype are borne on a 180-kb plasmid, the extrachromosomal element(s) do not confer a fitness cost probably due to a presence of siderophore coding genes and/or other factors that ensure plasmid stability [12]. Resistance to $\beta$-Lactams, quinolones, aminoglycosides and sulphonamides is widespread in K. pneumoniae [7]. There was no change in the resistance of the mucoid strain in the presence of $\beta$-Lactam only (amoxicillin) and $\beta$-Lactam $+\beta$-Lactamase inhibitor-sulbactam (augmentin), the data suggest that mucoid (hv) strains express $\beta$-Lactamases that are not sensitive to the $\beta$-Lactamase inhibitor sulbactam. Affinities for the $\beta$-Lactamases to $\beta$-Lactam inhibitor vary among bacterial species and among the different subtypes of the $\beta$-Lactamases. Therefore, resistance to a member of a class could confer resistance to other members of the same class. Cross resistance is obtainable among the members of the quinolones, $\beta$-Lactams and tetracyclines class of antibiotics.

\section{Conclusion}

Mucoid (hv) strains of $K$. pneumoniae are well adapted to thrive in hospital/healthcare environment due to multiple and high resistance capacity it possesses to various antibiotics and fit to disseminate within the community to cause infections in spite of extrachromosomal element(s) encoding antibiotic resistance and accessory phenotype in the mucoid (hv) strains.

\section{References}

[1] Tsai, Y.K., Fung, C.P., Lin, J.C., Chen, J.H., Chang, F.Y., Chen, T.L., et al. (2011) Klebsiella pneumoniae Outer Membrane Porins OmpK35 and OmpK36 Play Roles in Both Antimicrobial Resistance and Virulence. Antimicrobial 
Agents and Chemotherapy, 55, 1485-1493. http://dx.doi.org/10.1128/AAC.01275-10

[2] Hennequin, C., Aumeran, C., Robin, F., Traore, O. and Forestier, C. (2012) Antibiotic Resistance and Plasmid Transfer Capacity in Biofilm Formed with a CTX-M-15-Producing Klebsiella pneumoniae Isolate. Journal of Antimicrobial Chemotherapy, 67, 2123-2130. http://dx.doi.org/10.1093/jac/dks169

[3] Lee, H.C., Chuang, Y.C., Yu, W.L., Lee, N.Y., Chang, C.M., Ko, N.Y., et al. (2006) Clinical Implications of Hypermucoviscosity Phenotype in Klebsiella pneumoniae Isolates: Association with Invasive Syndrome in Patients with Community-Acquired Bacteraemia. Journal of Internal Medicine, 259, 606-614. http://dx.doi.org/10.1111/j.1365-2796.2006.01641.x

[4] Yu, W.-L., Ko, W.-C., Cheng, K.-C., Lee, H.-C., Ke, D.-S., Lee, C.-C., et al. (2006) Association between rmpA and magA Genes and Clinical Syndromes Caused by Klebsiella pneumoniae in Taiwan. Clinical Infectious Diseases, 42, 1351-1358. http://dx.doi.org/10.1086/503420

[5] Kawai, T. (2006) Hypermucoviscosity: An Extremely Sticky Phenotype of Klebsiella pneumoniae Associated with Emerging Destructive Tissue Abscess Syndrome. Clinical Infectious Diseases, 42, 1359-1361. http://dx.doi.org/10.1086/503429

[6] Coque, T.M., Oliver, A., Pérez-Díaz, J.C., Baquero, F. and Cantón, R. (2002) Genes Encoding TEM-4, SHV-2, and CTX-M-10 Extended-Spectrum $\beta$-Lactamases Are Carried by Multiple Klebsiella pneumoniae Clones in a Single Hospital (Madrid, 1989 to 2000). Antimicrobial Agents and Chemotherapy, 46, 500-510. http://dx.doi.org/10.1128/AAC.46.2.500-510.2002

[7] Paterson, D.L., Ko, W.-C., Von Gottberg, A., Mohapatra, S., Casellas, J.M., Goossens, H., et al. (2004) Antibiotic Therapy for Klebsiella pneumoniae Bacteremia: Implications of Production of Extended-Spectrum Beta-Lactamases. Clinical Infectious Diseases, 39, 31-37. http://dx.doi.org/10.1086/420816

[8] Yong, D., Toleman, M.A., Giske, C.G., Cho, H.S., Sundman, K., Lee, K., et al. (2009) Characterization of a New Metallo-Beta-Lactamase Gene, Bla(NDM-1), and a Novel Erythromycin Esterase Gene Carried on a Unique Genetic Structure in Klebsiella pneumoniae Sequence Type 14 from India. Antimicrobial Agents and Chemotherapy, 53, 5046-5054. http://aac.asm.org/cgi/content/long/53/12/5046

[9] Paterson, D.L., Mulazimoglu, L., Casellas, J.M., Ko, W.C., Goossens, H., Von Gottberg, A., et al. (2000) Epidemiology of Ciprofloxacin Resistance and Its Relationship to Extended-Spectrum Beta-Lactamase Production in Klebsiella pneumoniae Isolates Causing Bacteremia. Clinical Infectious Diseases, 30, 473-478. http://dx.doi.org/10.1086/313719

[10] Beceiro, A., Tomás, M. and Bou, G. (2013) Antimicrobial Resistance and Virulence: A Successful or Deleterious Association in the Bacterial World? Clinical Microbiology Reviews, 26, 185-230. http://dx.doi.org/10.1128/CMR.00059-12

[11] Hombach, M., Bloemberg, G.V. and Bo, E.C. (2011) Effects of Clinical Breakpoint Changes in CLSI Guidelines 2010 /2011 and EUCAST Guidelines 2011 on Antibiotic Susceptibility Test Reporting of Gram-Negative Bacilli. 1-11.

[12] Siu, L.-K.K., Huang, D.B. and Chiang, T. (2014) Plasmid Transferability of KPC into a Virulent K2 Serotype Klebsiella pneumoniae. BMC Infectious Diseases, 14, 176. http://dx.doi.org/10.1186/1471-2334-14-176 http://www.pubmedcentral.nih.gov/articlerender.fcgi?artid=3976155\&tool=pmcentrez\&rendertype=abstract

\section{Submit or recommend next manuscript to SCIRP and we will provide best service for you:}

Accepting pre-submission inquiries through Email, Facebook, Linkedin, Twitter, etc

A wide selection of journals (inclusive of 9 subjects, more than 200 journals)

Providing a 24-hour high-quality service

User-friendly online submission system

Fair and swift peer-review system

Efficient typesetting and proofreading procedure

Display of the result of downloads and visits, as well as the number of cited articles

Maximum dissemination of your research work

Submit your manuscript at: http://papersubmission.scirp.org/ 